\title{
Fracture behavior of an austenitic stainless steel with nanoscale
}

\section{deformation twins}

\author{
L. Xiong ${ }^{\mathrm{a}}$, Z.S. You ${ }^{\mathrm{a}, \mathrm{b}}$ and L. Lu ${ }^{\mathrm{a}, *}$
}

${ }^{a}$ Shenyang National Laboratory for Materials Science, Institute of Metal Research,

Chinese Academy of Sciences, 72 Wenhua Road, Shenyang 110016, People's

Republic of China

${ }^{\mathrm{b}}$ Herbert Gleiter Institute of Nanoscience, Nanjing University of Science and Technology, 200 Xiaolingwei Street, Nanjing 210094, People's Republic of China

* Corresponding author. E-mail addresses: 1lu@imr.ac.cn 


\begin{abstract}
A bulk nanotwinned austenitic stainless steel containing a large volume fraction of deformation twins was produced by dynamic plastic deformation. The tensile tests and $J$-integral fracture toughness measurements indicate that this steel exhibits a combination of high strength $\left(\sigma_{\mathrm{ys}}=920 \mathrm{MPa}\right)$ and considerable fracture toughness $\left(K_{\mathrm{JC}}>\right.$ $\left.126 \mathrm{MPa} \mathrm{m}{ }^{1 / 2}\right)$. The fracture is associated with localized shear band induced destruction of the nanotwinned structure and with micro-void development at the generated nanoscale large angle grain boundaries within the shear bands, which consumes large plastic energy and contributes the fracture resistance.
\end{abstract}

Keywords: Nanotwinned stainless steel; Fracture toughness; Shear deformation; Void nucleation 
Materials with embedded nanoscale twins have attracted extensive interest in recent years due to their novel mechanical properties, such as an unusual combination of high strength and good tensile ductility, enhanced strain hardening capacity, and improved fatigue resistance [1-6]. These properties have an important bearing on the special characteristics of twin boundaries (TBs) that not only restrict dislocation propagation, but also react with and accumulate dislocations [3, 5]. Nanotwinned materials hold promise for applications as structural components, in which a fracture mechanics evaluation on their fracture resistance is highly demanded to ensure structural reliability and safety. However, in contrary to the strengthening and deformation mechanisms that have been extensively studied [7-10], investigations regarding the fracture mechanism of nanotwinned metals are extremely scarce.

The fatigue crack growth tests by Singh et al. [6] revealed that ultrafine-grained $\mathrm{Cu}$ with nanoscale twins exhibits enhanced damage tolerance in comparison to twinfree samples. Through in-situ transmission electron microscope (TEM) investigations, Shan et al. [11] observed that crack grew in a zig-zag path across nano-twin lamellae. The molecular dynamic (MD) simulations by Zeng et al. [12] and by Kim et al. [13] further demonstrated that nanoscale twins not only substantially blunted micro-crack tips, but also served as crack bridging ligaments as the crack advanced. These experimental investigations and MD simulations indicate that the incorporation of highdensity nanoscale twins is potentially beneficial to provide enhanced damage tolerance [2, 6, 12-15].

However, the studies carried out so far majorly focus on the fracture process of 
nanotwinned thin sheets that are inevitably in plane stress state, which may be distinctly different from the fracture behavior of bulk samples in plane strain state. Accurate evaluation of the intrinsic plain strain fracture toughness of nanotwinned materials, which enables the damage tolerant design required for many structural applications, is still lacking. The scarcity of such researches can be in part ascribed to the fact that current preparation techniques, such as electrodeposition and magnetron sputtering [4, $16,17]$, do not deliver adequate sample volumes for fracture toughness tests.

Contrarily to growth nano-twins, nanoscale deformation twins are more easily generated during plastic deformation of metals with medium or low stacking fault energies, which makes it feasible to prepare bulk nanotwinned metals [18-20]. For instance, dynamic plastic deformation (DPD) has been demonstrated to be a practical approach to introduce nanotwinned structures in various kinds of bulk metals and alloys [21-24]. In the present study, a large volume fraction ( $60 \%)$ of nanoscale deformation twins is introduced into a $316 \mathrm{~L}$ stainless steel (SS) by DPD under a controlled compression strain. The fracture toughness of this bulk nanotwinned steel is then evaluated by elastic-plastic $J$-integral method, and the fracture process is discussed based on microstructural characterization and fractographic analysis.

The material used is a commercial AISI 316L SS with a composition of Fe-16.42Cr0.02C-0.37Si-1.42Mn-0.011S-0.040P (wt.\%). The as-received steel was first annealed at $1200{ }^{\circ} \mathrm{C}$ for one hour to generate uniform equiaxed austenitic grains with an average size of $\sim 100 \mu \mathrm{m}$, and then treated by the DPD technique at ambient temperature. The setup and processing parameters of the DPD treatment were described in detail 
elsewhere [25]. Cylindrical samples with a diameter of $23 \mathrm{~mm}$ and a height of $4.5 \mathrm{~mm}$ were compressed into disks with final dimensions of $28 \mathrm{~mm}$ in diameter and $3 \mathrm{~mm}$ in height. Accordingly, the imposed total true strain, defined as $\varepsilon=\ln \left(h_{\mathrm{i}} / h_{\mathrm{f}}\right)$, is about 0.4 , where $h_{\mathrm{i}}$ and $h_{\mathrm{f}}$ are the initial and final height of the treated sample, respectively. This strain was selected in purpose to make the volume fraction of deformation twins as large as possible.

The fracture toughness were assessed by $J$-integral measurement using a single specimen procedure according to ASTM E1820-11 [26]. For the $J$-integral test, miniaturized single edge-notched bend (SEB) specimen with a thickness of $2 \mathrm{~mm}$, a width of $4 \mathrm{~mm}$, a span distance of $16 \mathrm{~mm}$ and a total length of $22 \mathrm{~mm}$, was machined from the DPD disk, with the thickness direction of the SEB specimen corresponding to that of the DPD disk. The crack plane normal direction and the crack propagation direction are parallel to the tangential and radial directions of the DPD disk, respectively. The SEB specimens were first notched by electro-discharge machine and then fatigue pre-cracked to a total original crack length $a_{0}$ of $\sim 2 \mathrm{~mm}$. Finally, the samples were monotonically bent to extend the sharp crack on an Instron 5848 micro-tester at a constant displacement rate of $\sim 0.3 \mathrm{~mm} \mathrm{~min}^{-1}$. The corrected cross-head displacement of the tensile machine was used to represent the load-line displacement $v$ [27], and the instantaneous crack length $a$ was monitored using direct current potential drop method [28]. With the synchronously recorded $P, v$, and $a$, the $J$-integral resistance curve was computed following the standardized procedure [26].

To reveal the fracture process, the microstructure of the as-treated and fracture 
tested DPD sample was characterized by a scanning electron microscope (SEM; FEI NovaNano 430) and a TEM (JEOL 2010). The fracture surface was examined by the SEM, and by a confocal laser scanning microscope (CLSM; Olympus LEXT OLS4000) to determine the three-dimensional topography.

Cross-sectional SEM observations of the DPD sample (Fig. 1a) indicate that the grain boundaries (GBs) are still distinguishable and the original equiaxed grains become slightly elongated normal to the compression direction. The transverse grain size ranges from $20 \mu \mathrm{m}$ to $100 \mu \mathrm{m}$, with an average value of $50 \mu \mathrm{m}$. Numerous deformation bands making angles close to $\pm 45^{\circ}$ with respect to the DPD compression direction can be detected in most grains. Closer TEM observations (Fig. 1b) reveal that these bands are deformation twins, verified by the corresponding selected area electron diffraction (SAED) pattern. Most of the twin/matrix lamellae are thinner than $200 \mathrm{~nm}$ and the average thickness is $\sim 40 \mathrm{~nm}$. Inside the twin/matrix lamellae, there are numerous dislocations accumulating at the TBs. Statistical analysis indicates that more than $60 \%$ of the deformed grains possess such deformation twins. No shear bands or cracks were detected due to the small imposed strain. Besides the grains with deformation twins, there are also some deformed grains with only dislocation tangles and/or dislocation cells, see Fig. 1c and the single crystal SAED pattern, corresponding to the featureless areas in Fig. 1a. These twin-free grains were found to disperse among the nanotwinned grains and to occupy the remaining $40 \%$ volume.

Tensile tests show that the as-annealed coarse-grained 316L SS possesses a yield strength $\sigma_{\mathrm{ys}}$ of $275 \pm 5 \mathrm{MPa}$ and an ultimate tensile strength $\sigma_{\mathrm{uts}}$ of $585 \pm 9 \mathrm{MPa}$. After 
the DPD treatment, $\sigma_{\mathrm{ys}}$ is increased to $920 \pm 20 \mathrm{MPa}$ and $\sigma_{\mathrm{uts}}$ to $962 \pm 20 \mathrm{MPa}$. Both $\sigma_{\mathrm{y}}$ and $\sigma_{\mathrm{uts}}$ are remarkably improved as compared to those of the coarse-grained counterpart, which has been demonstrated to arise out of the nanoscale deformation twins impeding dislocation movement $[22,23]$. In contrary, the DPD treatment results in obvious reduction in tensile ductility. The uniform elongation $\varepsilon_{\mathrm{u}}$ decreases from 58 $\pm 5 \%$ at the coarse-grained state to only about $1 \pm 0.5 \%$, and the elongation to failure $\varepsilon_{\mathrm{f}}$ is reduced from $67 \pm 4 \%$ to $15 \pm 1 \%$.

Fig. 2a shows the representative curves of the force $P$ and the instantaneous electric resistance $R$ as a function of load-line displacement $v$ for the DPD sample. $R$ values were used to calculate the crack extension $\Delta a$ during bending by using a calibrated $R-$ $a$ curve. The calculated $J-\Delta a$ curves (Fig. $2 b$ ) indicate that the DPD sample exhibits stable crack growth and the crack propagation resistance significantly increases as the crack extends, analogous to the fracture tests of other nanostructured metals [29-31]. The crack blunting line is represented by $J=2 \sigma_{\mathrm{Y}} \Delta a$, where the effective yield strength $\sigma_{\mathrm{Y}}=941 \mathrm{MPa}$ is the average of $\sigma_{\mathrm{ys}}$ and $\sigma_{\mathrm{uts}}$. The intersection of the $0.2 \mathrm{~mm}$ offset blunting line with the $J-\Delta a$ curve yields a provisional $J$-integral value $J_{\mathrm{Q}}$ of $148 \pm 7 \mathrm{~kJ}$ $\mathrm{m}^{-2}$, which corresponds to the $J$-integral as the crack extends $0.2 \mathrm{~mm}$. The sample geometry satisfies the minimum thickness requirement for obtaining a size-independent value of fracture toughness, namely $B \geq 10 J_{\mathrm{Q}} / \sigma_{\mathrm{Y}}$ [26]; however, the straight crack front requirement is violated as the crack front becomes curved due to significant confinement from plane stress side surfaces of miniaturized samples. As a consequence, the present $J_{\mathrm{Q}}$ of DPD 316L SS should only be regarded as the upper limit of plane- 
strain fracture toughness $J_{\mathrm{IC}}$.

Alternatively, the $J$-integral of stable crack growth initiation can be estimated by measuring the crack tip opening displacement $\delta$. Fig. 3a displays the SEM observation on the transition area from the fatigue pre-crack region to the overload fracture area in the sample center (in plane strain state), where an obvious stretched zone can be detected (marked between the two white dash lines). The CLSM measurements on the stretched zone show that the fracture initiation site is $20 \pm 5 \mu \mathrm{m}$ high with respect to the fatigue pre-crack plane, an indication of significant plastic blunting of the crack tip prior to the initiation of crack extension. To further visualize this obvious crack blunting, a sample bent to the critical point at which crack growth initiates were sectioned at the midpoint along sample thickness and observed by SEM. The photo in Fig. $3 \mathrm{~b}$ confirms that the crack has been evidently blunted to a root radius of $18 \pm 3 \mu \mathrm{m}$, consistent with the stretched zone height. Based on these measurements, $\delta$ at crack initiation is estimated to be $\sim 40 \mu \mathrm{m}$ (sum of the stretch zone heights at the two halves of a fractured specimen or two times of the crack tip root radius). The crack initiation $J$-integral $J_{\mathrm{i}}$, calculated by using the relationship $J_{\mathrm{i}}=2 \sigma_{\mathrm{Y}} \delta$ [32], is $75 \mathrm{~kJ} \mathrm{~m}^{-2} . J_{\mathrm{IC}}$ is defined as $J$ integral at $0.2 \mathrm{~mm}$ crack extension and hence should be larger than $J_{\mathrm{i}}$, since $J$-integral increases with crack growth.

Combining the $J-\Delta a$ curve and the fractographic measurement, the valid $J_{\text {IC }}$ should fall in the range of 75 to $148 \mathrm{~kJ} \mathrm{~m}^{-2}$. The equivalent critical stress-intensity factor $K_{\mathrm{JC}}$ is calculated as 126 to $177 \mathrm{MPa} \mathrm{m}^{1 / 2}$ by using the relationship $K_{\mathrm{JC}}=\sqrt{E J_{\mathrm{IC}} /\left(1-v^{2}\right)}$, where $E$ is the Young's modulus and $v$ is the Poisson's ratio. For comparison, the 
fracture toughness of the coarse-grained austenitic stainless steel with low $\sigma_{\mathrm{ys}}$ is reported to be in the range of $175-400 \mathrm{MPa} \mathrm{m}^{1 / 2}$ [33], while conventional cold working reduces the fracture toughness to $85 \mathrm{MPa} \mathrm{m}^{1 / 2}$ as the $\sigma_{\mathrm{ys}}$ is increased to $725 \mathrm{MPa}$ [34]. The results demonstrate that the nanoscale TBs can remarkably improve the strength of 316L SS while maintaining considerable fracture toughness.

Fractographic observations (Fig. 3c) show homogeneous equiaxed dimples, manifesting that the sample fails in a ductile manner involving void nucleation and coalescence. The diameters of most dimples are larger than $20 \mu \mathrm{m}$, with an average value of $\sim 48 \mu \mathrm{m}$, which is close to the grain size $(\sim 50 \mu \mathrm{m})$ but nearly three orders of magnitude larger than the average twin thickness. Three-dimensional (3D) topography (Fig. 3d) obtained by CLSM of the same area in Fig. 3c demonstrates that these dimples appear not to lie in a common plane. The peak-to-valley height $\Delta h$ of the selected area can be as large as $230 \mu \mathrm{m}$, much larger than the grain size.

For the crack propagating in a ductile coarse-grained material with low yield strength, void formation and coalescence associated with the presence of non-metallic inclusions are commonly observed $[35,36]$. In this study, the $316 \mathrm{~L}$ SS sample had a high purity level and was carefully heat treated, and hence the inclusion density is relatively low. Some coarse dimples on the fracture surface were characterized to be nucleated at these inclusions (with inclusions being detected at the dimple bottoms). The effects of the inclusions in the nanotwinned $316 \mathrm{~L} \mathrm{SS}$ on the void nucleation and on the significant crack path tortuosity are analogous to those in conventional ductile fracture. However, in addition to the dimples nucleated at the inclusions, there are still 
a large amount of inclusion-free dimples with the size of several tens of micrometer on the tortuous fracture surface, an indication that the fracture of nanotwinned 316L SS is quite different from that of conventional coarse-grained steels.

In order to unveil the intrinsic fracture mechanism, the microstructural evolution ahead the crack tip was carefully examined by SEM and TEM, as shown in Figs. 4. Fig. 4a shows numerous micro-voids are formed in the plastic zone ahead of the blunted crack tip. Closer view (Fig. 4b) on the microstructure adjacent to a micro-void clearly indicates that evident strain localization in form of shear bands (marked by white arrows) develops in the nanotwinned grain and that the void is located in the middle of a welldeveloped shear band with a width of $\sim 2 \mu \mathrm{m}$. It appears that the nucleation of microvoid in the nanotwinned structure is intimately associated with the occurrence of localized shear bands, which has been recognized to control the failure of some metals [37].

Fig. $4 \mathrm{c}$ shows several freshly nucleated micro-bands with a thickness of $\sim 100 \mathrm{~nm}$. These bands accommodate the plastic deformation when dislocation activities are suppressed by the high-density TBs and gradually develop into shear-bands with increasing strain [38]. The nanotwinned structure inside the shear bands is dramatically damaged by the substantial localized plastic deformation and eventually converted to elongated sub-grains. The transverse thickness of the sub-grains is $\sim 50 \mathrm{~nm}$ inside the shear bands, as shown in Fig. 4d. The SAED pattern from the area outlined by the dashed circle displays diffraction spots in arc shape, manifesting that high-angle GBs have formed inside the well-developed shear bands. Under further accumulated strain, 
the nanoscale GBs or triple junctions probably directly act as nucleation sites for microvoids inside the shear band, because of their high energy states and the buildup of stress concentration caused by plastic strain incompatibility between neighboring grains $[39$, 40].

The above microstructural observations demonstrate that void nucleation through localized shear deformation and complete destruction of nanotwins (as displayed in Fig. 4) should be a possible and important underlying mechanism. This process produces the dimples on the tortuous fracture surface (Fig. 3c). Both the void nucleation and interconnection involve extensive deformation and damage of the nanotwins, thus consuming large irreversible plastic energy. During these processes, it is worth noting that the high-density coherent TBs do not directly act as void nucleation sites, and hence the average dimple size is still quite large, close to the mean grain diameter (Fig.3c). This feature is fundamentally distinct from the traditional strengthening approaches. For instance, in materials strengthened by grain refinement or second-phase particles, the dimple sizes become smaller, and the crack path tortuosity is largely reduced as well, due to the increased density of void nucleation sites incorporated by such strengthening agents $[41,42]$, resulting in reduced fracture resistance. In contrary, the nanotwinned structure is unique in strengthening the materials significantly while not dramatically deteriorating the fracture toughness.

As summary, the fracture behavior of a nanotwinned 316L SS containing $60 \%$ volume fraction of randomly orientated nanotwinned grains was examined. The results indicate that a combination of high tensile strength and considerable fracture toughness 
can be achieved in the nanotwinned 316L SS. The microstructural characterization near the crack tip demonstrates that the void nucleation and crack propagation are dominated by the localized shear deformation destructing the nanoscale twins into largely misoriented GBs. This process dissipates large irreversible plastic energy, and appears to be beneficial for providing crack propagation resistance.

\section{Acknowledgements}

The authors acknowledge financial support from the National Basic Research Program of China (973 Program, 2012CB932202), the National Science Foundation of China (Grant Nos. 51420105001, 51171181, 51371171, 51471172 and 51401211).

\section{References}

[1] L. Lu, Y.F. Shen, X.H. Chen, L.H. Qian, K. Lu, Science, 304 (2004) 422-426.

[2] L. Lu, X. Chen, X. Huang, K. Lu, Science, 323 (2009) 607-610.

[3] K. Lu, L. Lu, S. Suresh, Science, 324 (2009) 349-352.

[4] Y.F. Shen, L. Lu, Q.H. Lu, Z.H. Jin, K. Lu, Scr. Mater., 52 (2005) 989-994.

[5] L. Lu, Z.S. You, K. Lu, Scr. Mater., 66 (2012) 837-842.

[6] A. Singh, L. Tang, M. Dao, L. Lu, S. Suresh, Acta Mater., 59 (2011) 2437-2446.

[7] J.A. Brown, N.M. Ghoniem, Acta Mater., 57 (2009) 4454-4462.

[8] Z.H. Jin, P. Gumbsch, K. Albe, E. Ma, K. Lu, H. Gleiter, Acta Mater., 56 (2008) $1126-1135$.

[9] Z. You, X. Li, L. Gui, Q. Lu, T. Zhu, H. Gao, L. Lu, Acta Mater., 61 (2013) 217- 
[10] T. Zhu, H. Gao, Scr. Mater., 66 (2012) 843-848.

[11] Z.W. Shan, L. Lu, A.M. Minor, E.A. Stach, S.X. Mao, JOM, 60 (2008) 71-74.

[12] Z. Zeng, X. Li, L. Lu, T. Zhu, Acta Mater., 98 (2015) 313-317.

[13] S.-W. Kim, X. Li, H. Gao, S. Kumar, Acta Mater., 60 (2012) 2959-2972.

[14] H. Zhou, S. Qu, Nanotechnology, 21 (2010) 035706.

[15] H.F. Zhou, S.X. Qu, W. Yang, Model. Simul. Mater. Sci. Eng., 18 (2010).

[16] A.M. Hodge, Y.M. Wang, T.W. Barbee, Jr., Scr. Mater., 59 (2008) 163-166.

[17] X. Zhang, A. Misra, H. Wang, M. Nastasi, J. Embury, Appl. Phys. Lett., 84 (2004) 1096-1098.

[18] M.A. Meyers, O. Vohringer, V.A. Lubarda, Acta Mater., 49 (2001) 4025-4039.

[19] E.H. Lee, T.S. Byun, J.D. Hunn, M.H. Yoo, K. Farrell, L.K. Mansur, Acta Mater., 49 (2001) 3269-3276.

[20] E.H. Lee, M.H. Yoo, T.S. Byun, J.D. Hunn, K. Farrell, L.K. Mansur, Acta Mater., 49 (2001) 3277-3287.

[21] Y.S. Li, N.R. Tao, K. Lu, Acta Mater., 56 (2008) 230-241.

[22] F.K. Yan, G.Z. Liu, N.R. Tao, K. Lu, Acta Mater., 60 (2012) 1059-1071.

[23] H.T. Wang, N.R. Tao, K. Lu, Acta Mater., 60 (2012) 4027-4040.

[24] Y. Zhang, N.R. Tao, K. Lu, Acta Mater., 59 (2011) 6048-6058.

[25] N.R. Tao, K. Lu, J. Mater. Sci. Technol., 23 (2007) 771-774.

[26] Standard Test Method for Measurement of Fracture Toughness. Annual Book of ASTM Standards. ASTM E1820-11: American Society for Testing and Materials, 
Philadelphia, PA, USA, 2011.

[27] M.T. Kirk, E.M. Hackett, Fracture Mechanics:Eighteenth Syposium, ASTM STP 945.

[28] H.H. Johnson, Mater. Res. Stand., 5 (1965) 442-445.

[29] A. Hohenwarter, R. Pippan, Mater. Sci. Eng. A, 540 (2012) 89-96.

[30] A. Hohenwarter, R. Pippan, Acta Mater., 61 (2013) 2973-2983.

[31] I. Sabirov, R.Z. Valiev, I.P. Semenova, R. Pippan, Metall. Mater. Trans. A, 41 (2010) 727-733.

[32] T.L. Anderson, Fracture Mechanics:Fundamentals and Applications, CRC Press, Boca Raton, 1994.

[33] W.J. Mills, Int. Mater. Rev., 42 (1997) 45-82.

[34] J.E. Pawel, J. Nucl. Mater., 212-215 (1994) 442-447.

[35] M.F. Ashby, Philos. Mag., 21 (1970) 399-424.

[36] W.J. Mills, Eng. Fract. Mech., 30 (1988) 469-492.

[37] Q. Xue, M.A. Meyers, V.F. Nesterenko, Acta Mater., 50 (2002) 575-596.

[38] Q. Xue, E.K. Cerreta, G.T. Gray III, Acta Mater., 55 (2007) 691-704.

[39] A. Hohenwarter, R. Pippan, Scr. Mater., 64 (2011) 982-985.

[40] K.S. Kumar, H. Van Swygenhoven, S. Suresh, Acta Mater., 51 (2003) 5743-5774.

[41] A. Hohenwarter, R. Pippan, Phil. Trans. R. Soc. A, 373 (2015).

[42] G. Lacroix, T. Pardoen, P.J. Jacques, Acta Mater., 56 (2008) 3900-3913. 


\section{Figure Captions}

Figure 1. (a) SEM observations of the cross-sectional microstructure of the DPD sample, showing numerous deformation bands inside most of the original coarse grains. (b) and (c) TEM images of the deformation twins and the deformed coarse grains, corresponding to $\mathrm{b}$ and $\mathrm{c}$ regimes in (a), respectively.

Figure 2. (a) Typical curves of force $P$ and electrical resistance $R$ versus load-line displacement $v$ for the fracture test of DPD 316L SS sample. (b) Calculated $J$ - $\Delta a$ curve from the data in (a). The inset in (b) displays the photo of SEB samples before and after the bending test.

Figure 3. (a) SEM observations on the transition area from the fatigue pre-crack to the overload fracture, which clearly shows the presence of a stretched zone, marked by white dash lines. (b) Cross-sectional observation of the main crack tip at the midsection of SEB sample unloaded near the peak load. (c) Closer observations on the fracture surface showing typical plastic dimples. (d) Three-dimensional CLSM observations on the area corresponding to Fig. 3c, depicting the depth variations of the fracture surface. Color in the color-bar represents the relative depth in unit of $\mu \mathrm{m}$.

Figure 4. Microstructural evolution ahead the crack tip observed at the midsection of a SEB sample unloaded near the peak load during bending. (a) Blunted crack tip and numerous micro-voids near the crack tip. (b) Microstructure near a micro-void, showing that multiple shear bands cut through the TBs and the micro-void is located inside a 
shear band. (c) TEM observations showing several micro-bands in the nanotwinned area. (d) Nanoscale grains with relatively large misorientations within a thick shearband. 

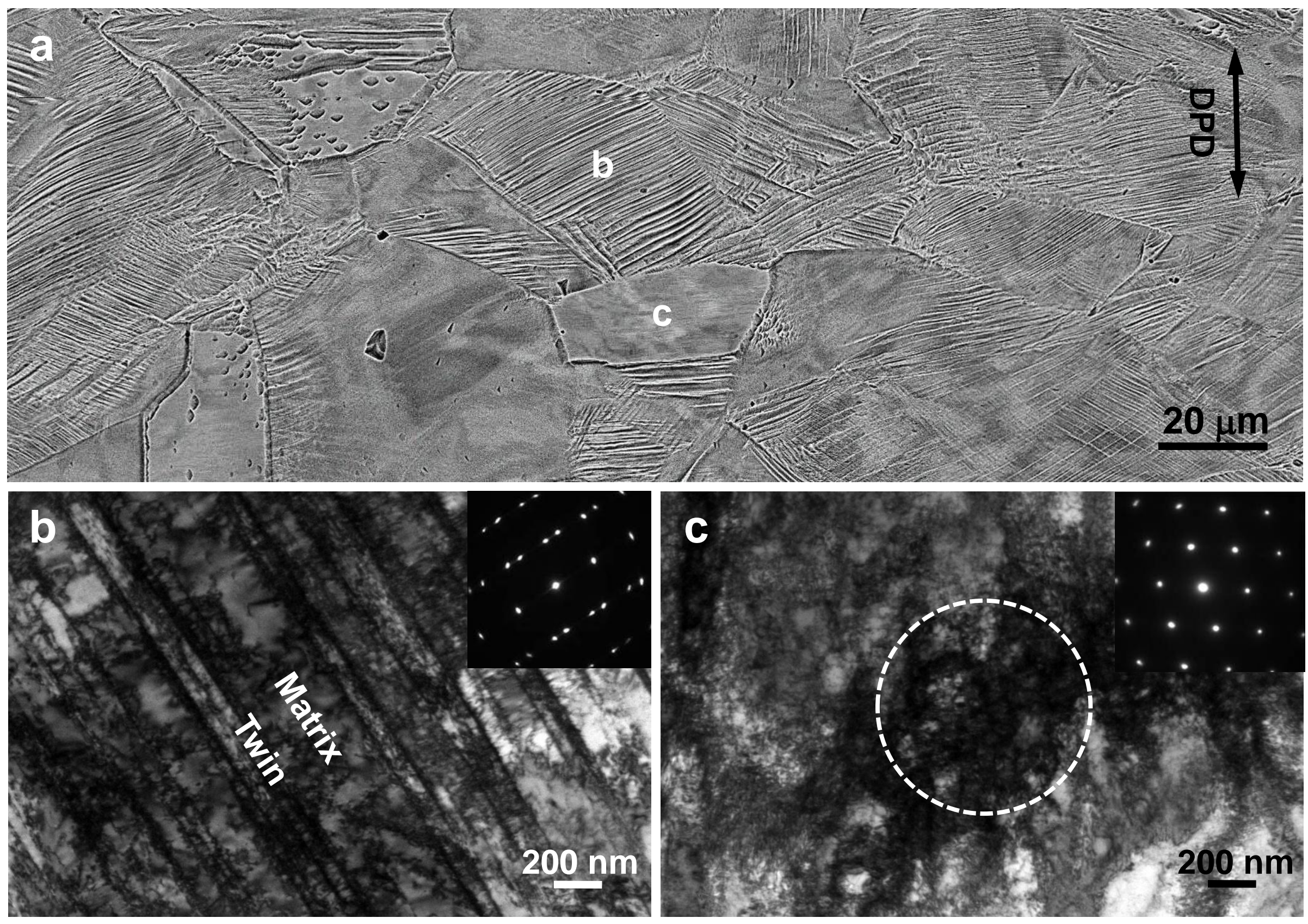

Fig. 1 /Xiong et al 

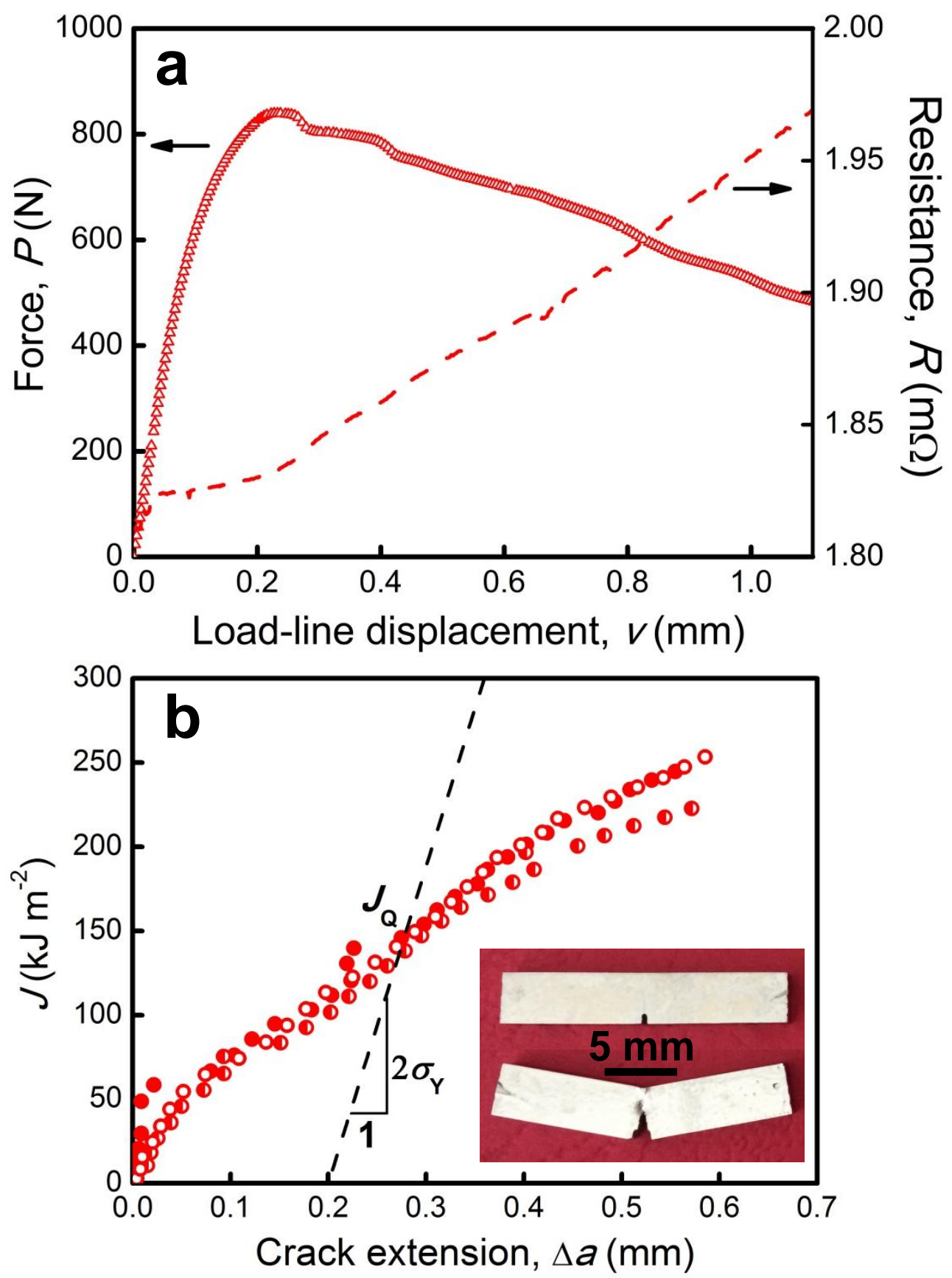

Fig. 2 /Xiong et al 

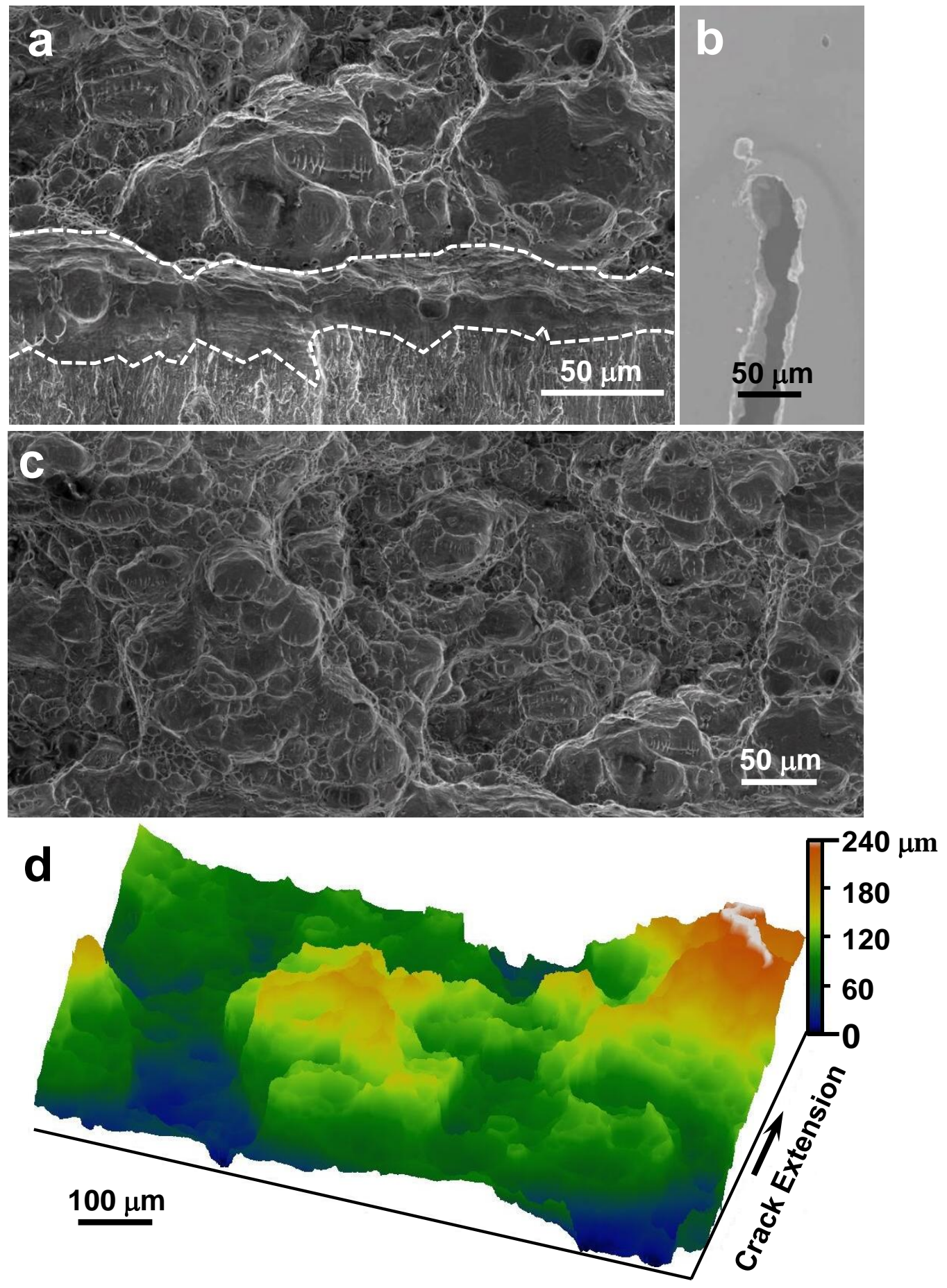

Fig. 3 /Xiong et al 

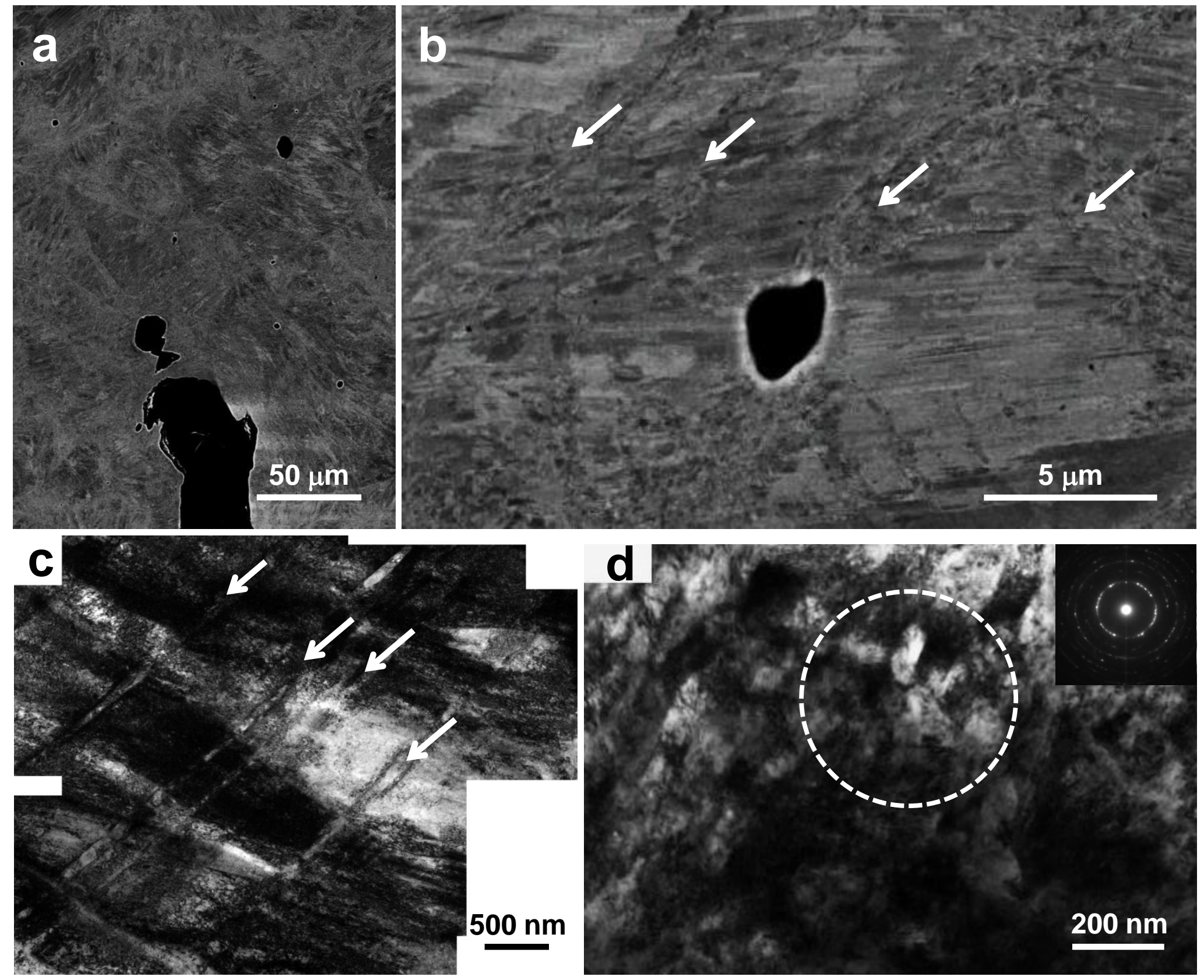

Fig. 4 /Xiong et al 


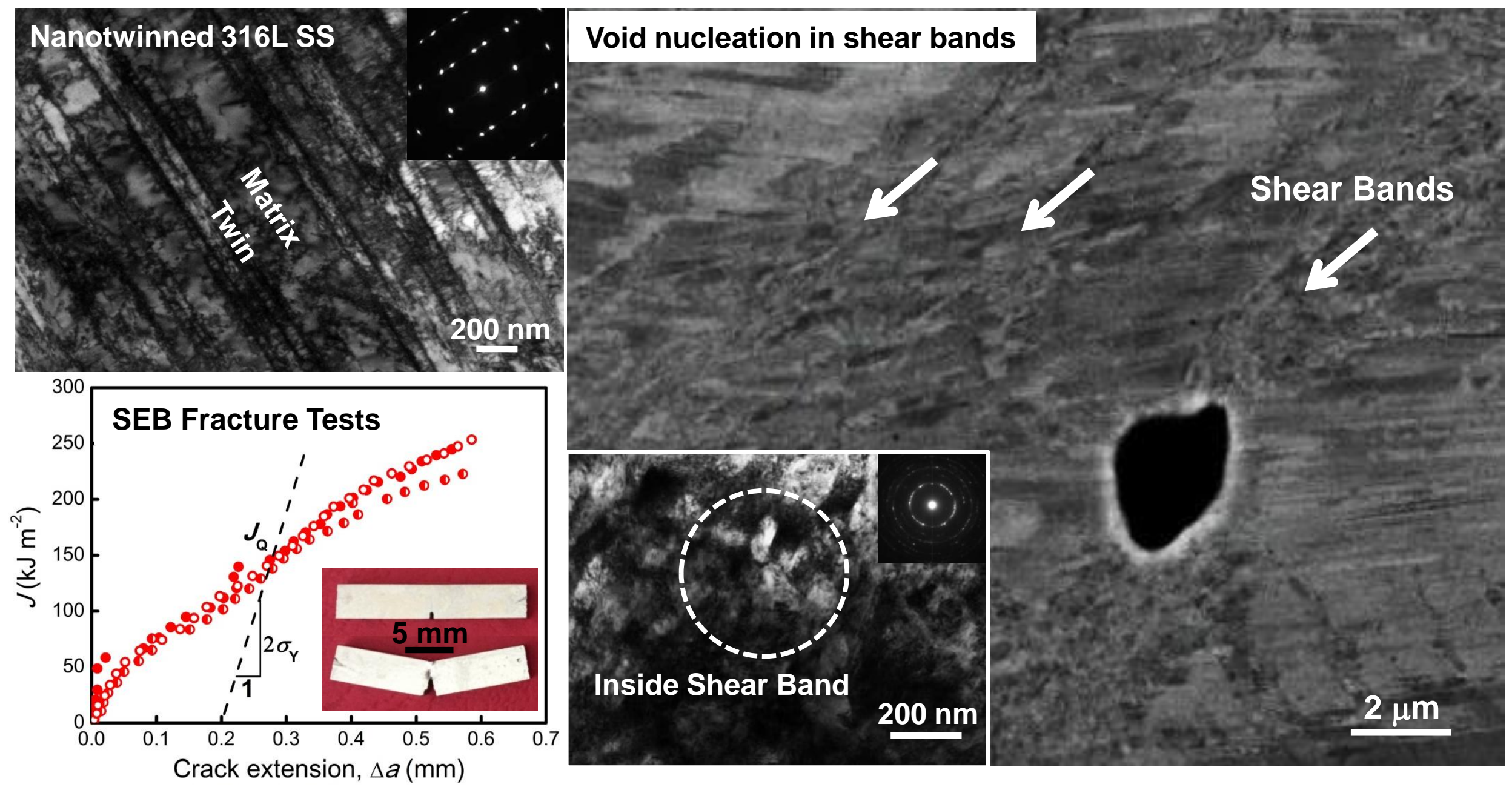

Nanotwin strengthened stainless steel exhibits high tensile strength and considerable fracture toughness. The dominant fracture of this steel is associated with the void nucleation in nanograin areas originated from the destruction of TBs via localized shear deformation. 